\title{
Design as Means of Countering Vandalism
}

\author{
Sokolov A ${ }^{\mathrm{a}}$, Kukhta $\mathrm{M}^{\mathrm{a} *}$, Kornienko $\mathrm{M}^{\mathrm{a}}$, Kondratyeva $\mathrm{Y}^{\mathrm{a}}$, Kukhta $\mathrm{A}^{\mathrm{b}}$ \\ * Corresponding author: Kukhta M., eukuh@mail.tomsknet.ru \\ ${ }^{a}$ National Research Tomsk polytechnic university 634050, Lenina str., 30, Tomsk, Russia, E-mail: eukuh@mail.tomsknet.ru 8- \\ 913-107-51-24 \\ ${ }^{b}$ National Research Tomsk State University 634050, Lenina str.36, Tomsk, Russia, E-mail: artkuh@mail.tomsknet.ru, 8-913- \\ 877-96-36
}

\begin{abstract}
http://dx.doi.org/10.15405/epsbs.2017.01.53

In this research the design in hardening of aesthetically valuable objects against vandalism is analyzed. There are two distinguished forms of vandalism: meaningless and meaningful ones. The purpose of this research is to develop design methods that enhance protection of aesthetically valuable objects against vandals. The harmonious development of the society is the main goal of culture. Actions that prevent movement to the harmonious development of society are treated as vandalism in this study, in a broad sense of the word.

Meaningful vandalism is aimed at the destruction or desecration of objects with aesthetic or cultural value. The counteraction against the meaningful vandalism cannot be realized through the design. The research of vandalism motivation shows that meaningless vandalism does not have a pronounced goal. The main characteristic of meaningless vandalism is a destruction of the most at the lowest cost. It is shown in the study, how to resist the meaningless vandalism by using the objects of design. The relationship of design and mindless vandalism is not linear. Inharmonious design can provoke this type of vandalism. It is important to avoid stress and attention concentrators, when designing aesthetically valuable objects.

Aimless (mindless) production is a kind of meaningless vandalism. Trash is the lower border of mindless vandalism and the upper border is meaningful vandalism. Violation of physical and spiritual ecology is the main source of mindless vandalism.
\end{abstract}

C 2017 Published by Future Academy www.FutureAcademy.org.uk

Keywords: Vandalism protection; design; aesthetically valuable objects; motivation.

\section{Introduction}

The present time is characterized by the increased vandalism. The development of measures to reduce it should be based on a steady scientific basis and this should be started with terminology. But even at this stage the researcher is faced with uncertainty. For example, in the Soviet Encyclopaedic 
Dictionary: "Vandalism is a meaningless destruction of cultural and material values" and in the French Encyclopaedic Dictionary "Larousse": "Vandalism is a state of mind that forces to destroy beautiful things such as works of art". Generalization of these two variants gives a third one - vandalism is a meaningless destruction of objects that have aesthetic value. The society aims to increase protection of aesthetically valuable objects (AVO) from vandals. In a great measure this problem can be solved by design.

The purpose of this research is to develop design methods that enhance protection for objects against vandals.

To achieve this goal following tasks had been solved. Firstly, factors which influence vandalism were analysed. Secondly, known methods of vandalism prevention were investigated. Thirdly, new approaches in design that increase the protection of objects from vandalism were generated.

\section{Materials \& methods}

During the research, historical-cultural and comparative analysis methods are used to investigate the origins and intentions of the vandalism problem. In order to solve the problems and develop proposals that prevent (or reduce) the extension of vandalism authors used engineering (design and conceptual) methods.

As the given definition of vandalism - the meaningless destruction of aesthetically valuable objects the emphasis is on the senseless of destruction. This approach is shared by many modern researchers that is reflected in the local dictionaries. The term "vandalism" in different nations is treated differently. For example, an English researcher A. Goldstein highlights the intention, destructiveness and ownership of decomposable object. He gives the definition: "Vandalism is an intentional act of destruction or causing damage to other's property". (Goldstein A., 1996) In this case the main point is the intentional destruction. Without clarifying the object of destruction the question of intentions is difficult to resolve. Therefore in many empirical studies intentional and unintentional destructions are not distinguished. However the question of intentionality can be solved due to specifying the object of vandalism. If the object of vandalism is the other's property, the destruction is carried out deliberately by a competitor. It is pertinent thesis of Roman jurisprudence - look who benefits. Thus due to the restriction of the vandalism object the concept of vandalism is specified. When formulate any term the attention should be paid to the purpose for which the term is introduced. For example in the XIX century the term "vandalism" was used to refer the destruction or damage caused to works of art and architectural monuments. Since then the term "vandalism" has begun to include more kinds of destruction. This has led to an erosion of the term's accuracy. However, if to emphasize the attention on the destruction of aesthetically valuable objects the term "vandalism" gets high accuracy and stability. It contributes to the exactness of the description for facts associated with the destruction of the aesthetic and cultural values.

In case of objects with aesthetic or cultural value the question of intentional or unintentional destruction is harder to resolve. Such objects serve utilitarian and also aesthetic and cultural function. Moreover aesthetic or cultural function is much more valuable than utilitarian. Satisfaction of aesthetic or cultural function is the responsibility of the whole society and so with the destruction of objects with 
aesthetic or cultural value the impact is applied to the whole of society. In this case it is more difficult to formulate the purpose of vandalism and to solve the question of intentional or unintentional destruction. From the standpoint of society the destruction of aesthetically valuable objects is senseless. Accordingly to this the destruction of such objects is vandalism. Some people in certain circumstances can have a mental condition that makes them destroy beautiful things. Such people are classified as deviant.They do so sometimes against the society's standards and these deviations may be widespread. The presence of mass character for any phenomenon indicates certain patterns that can be learned and used. The method of using laws found through psychology is considered productive. However, the results obtained with this method are ambiguous. This can be explained in the following way. If to consider that vandalism is simply a crime without taking into accounts the specifics of the destructed object the result of such research will be controversial. If to narrow the destructed object to the aesthetically valuable the goal will be automatically instantiated as the destruction of aesthetic value.

\section{Results \& discussion}

The definition of the term "vandalism" should be clarified again by using the concept of aesthetic value. The complexity of "aesthetic value" concept is shown even in Plato's dialogue "Hippias Major" (Plato, 1994). In fact the accuracy of this concept manifests itself in the context of a particular study. For example if there is a study of the function items any specific thing can be regarded as fine if it perfectly fulfills its function. Flatness and smoothness of the surface or shine and transparency of glass can be taken for aesthetic value. There is a certain category of people which can be irritated by smoothness of the surface or shine of glass and therefore they sometimes destroy things with such characteristics. This type of vandalism has no clearly defined target; it seems pointless so let's call it "meaningless vandalism" (without the quotes further).Vandalism that has a clearly expressed goal is radically different from that. An accurate definition of such vandalism was given by D.V. Zhmurov: "Vandalism is damaging actions aimed at the destruction, discrediting, desecration of the cultural values, which an individual, due to various reasons, perceives as hostile to itself (the social group to which he belongs)." (Zhmurov D., 2011) In this kind of vandalism the goal is clearly expressed and it points to meaningfulness of vandals' actions so let's call it "meaningful vandalism" (without the quotes further).

Meaningful and meaningless vandalisms have different goals. Therefore counteracting methods are fundamentally different for each type. The purpose of a meaningful vandalism is the destruction of the values of the specific culture which vandal perceives as hostile to him. Factually in meaningful vandalism individual (social group) is transformed into an instrument of culture's destruction. Therefore measures to counter meaningful vandalism are not in the field of technology for creating aesthetically valuable objects but in cultural protection. Properly speaking the path of counteracting meaningful vandalism does not pass through design which has structural purposes.

Meaningless vandalism has no clearly defined goals but has different motivations. The study of motivation allows us to develop methods to counteract this kind of vandalism. It is important to take into account here that the same needs can serve as a basis for constructive and deconstructive motives of behavior. Moreover it is not always possible to divide clearly the constructive and destructive effects 
of behavior. As an illustrative example, let's consider the following scenario. The monotony of the building facade (and even more so with the fence) causes irritation. To remove this annoyance, architects use articulation of the building facade. To remove the irritation from the monotony of concrete fences the youth uses graffiti. From the standpoint of fence installers the aesthetic value is a monotonous surface of reinforced concrete sections installed on the line. In this case application of chaotic graffiti on the monotonous surface is perceived as vandalism. From the viewpoint of bionic style adherents setting of reinforced concrete sections of the fence on line is a destruction of the environment, i.e. vandalism of this environment. The way out from this contradiction appears in the following direction. If an act serves for development of the society's majority, then it should be taken constructively. In the case, of reinforced concrete fence as a constructive can be considered an experiment that was conducted in Tomsk. The fence around the construction site as a field for creativity were granted to the fans of graffiti. The paints were given but the condition was also posed one author fills one section over the entire surface with a drawing. The result was an open-air gallery of pictures (Fig. 1). Moreover these figures fit in harmoniously with the environment. In this case we received a good combination: on the one hand, the building of the church among the trees and on the other - bionic ligature of graffiti that reminds interlocking of tree branches. The harmonic composition of objectscan often be achieved by means of the bionic design principles.

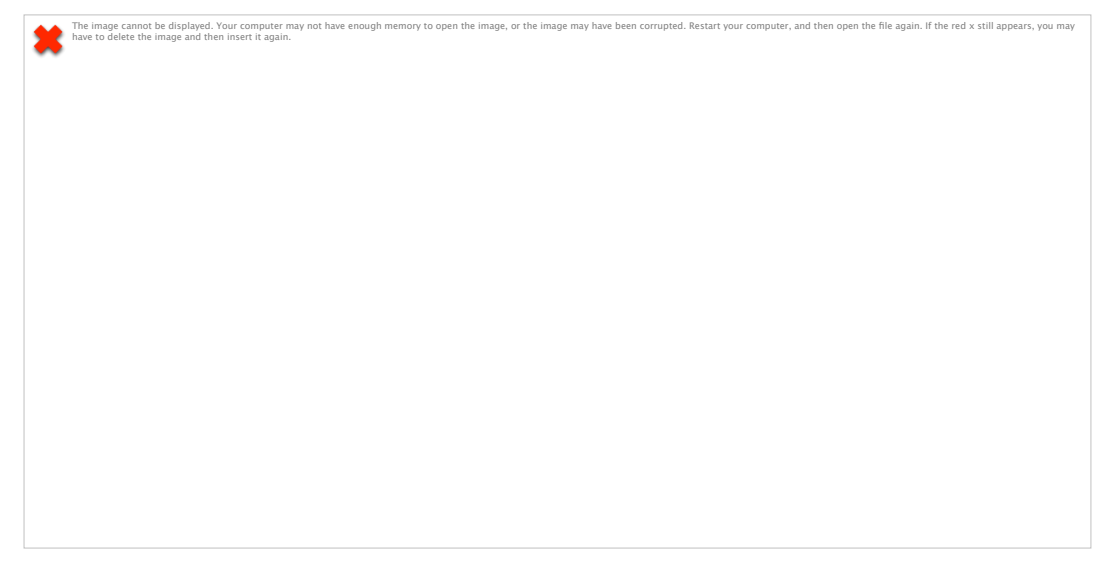

Fig. 1. The example of anti-vandalism

In this case there was a need to eliminate the irritation from the monotony of the fence. There were conditions, created for realization of the motive (search for identity). The result is an aesthetically valuable object; this is an example of anti-vandalism. Spontaneously it began to appear different ads at the same place, what disrupted the available aesthetic value (Fig. 2). This is a meaningless vandalism. It is notable that after graffiti, concentration of advertisements had increased in the corner joints of reinforced concrete sections. Before application that concentration was less pronounced. It has a convincing explanation. Before graffiti leaflets were visible anywhere on the surface of monotonous concrete sections. After graffiti information leaflets became visually lost in the background of pictures. Corners became the only place of attention so where newsletters are concentrated now. 


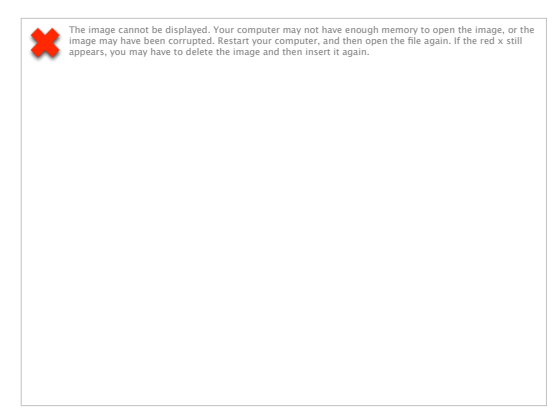

Fig. 2. Vandalism among anti-vandalism

This case shows that by means of design the reduction of the meaningless vandalism results can be achieved. One of the design tools is an attention management, so the object should be designed in a way that hubs of attention could easily be restored when they were violated. In the case of fence the good design course is to put the information stand on the corner.

Determination of vandalism motive is a complex issue, particularly in the meaningless vandalism. As a motive can be distinguished such cases as anger, boredom and study (Allen V., \& Greenberger D., 1978). There is even an aesthetic theory according to which the vandalism is considered from the standpoint of experimental aesthetics. However, the experimental results often have an ambiguous interpretation and theoretical generalization. For example, researchers (Allen V., \& Greenberger D., 1979) compared the pleasure obtained during the destruction of glass and wood. They came to the controversial conclusion that the glass is more pleasant to destroy because shapes of pieces are more complex and interesting. Perhaps more pleasure from destruction appears, if there are pieces of complex and interesting form. However, the obtained result on the glass cannot be transferred to a wood. When it comes to mindless vandalism, it is important to teenager to show "valiant prowess" with a single blow. The more the result of the destruction is, the more "prowess" it shows. Easier it can be achieved with glass partition, instead of scraping wood (it needs to tinker with). The thesis of mindless vandalism can be formulated as the destruction of the most at the lowest cost.

Implementation of this thesis may be illustrated by the following example. In Tomsk, there was a period of time when teenagers were twisting street benches (Fig. 3). Bench analysis shows a failure of its construction. Sidewall of the bench narrowed down to the base. At the point of contact with the ground, there were encountered tensions and that was used by vandals. As the fulcrum is narrowed, the bench could be easily shacked and twisted out of the ground - the maximum effect with the minimum of cost. When the base was extended (Fig. 4), the bench became both visually and physically more stable. In this case the tension concentrators were eliminated and so resistance to vandalism increased.

(Kukhta M., \&Sokolov A., 2014), (Sokolov A., \&Kukhta M. 2014). 


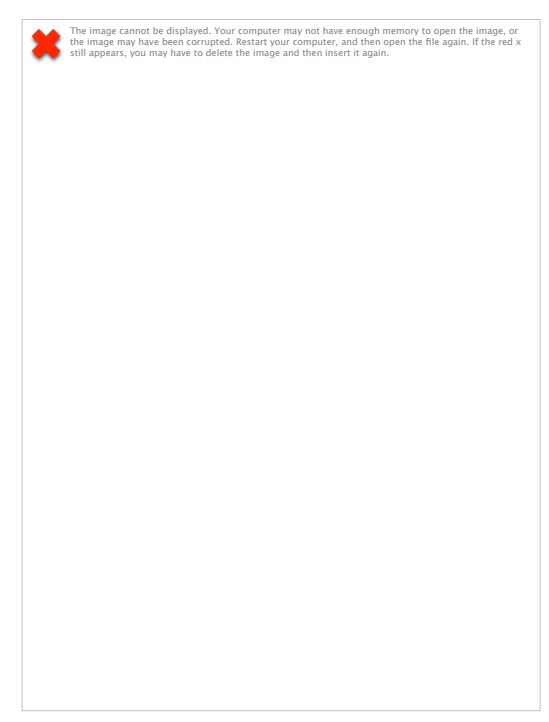

Fig. 3. A bench as the object of vandalism

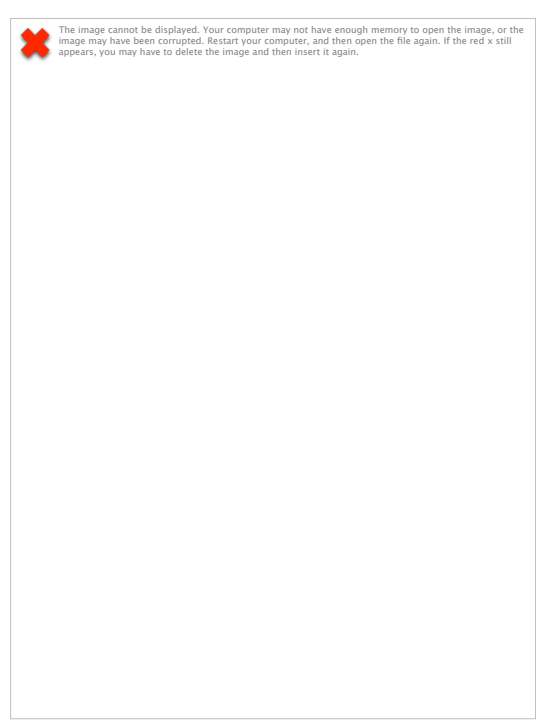

Fig. 4. Form correction

Thus we can formulate a thesis of counteracting mindless vandalism by means of design. The object should be designed to eliminate the stress concentrators and to have the appearance of physical strength. On the other hand physically durable construction can on the contrary give a rise for desire to destroy it. In this case we have a meaningful vandalism and the opposition to it comes out of the design scope.

Considering the thesis of meaningless vandalism (maximum destruction at minimum cost) fragile materials are often exposed to destruction and the glass in this regard is out of competition. It has intensified designers to search for aesthetic forms that obtained by the glass breaking. For example, a grid of cracks on the non-hardened glass obtained by a hit (Figure 5), can simulate the really observable phenomenon - the aura around the luminaire during the passage of the rays through the tree crown (Figure 6). (Kornienko M., \& Kukhta M., 2015). A grid of cracks on the hard-tempered glass (Fig. 7) can simulate a rete of streaks on the tree leaf. In both cases it turns into aesthetic form. It is complicated enough but can be obtained with one hit. It is noteworthy that an imitation of natural form has specific aesthetic value. Under certain conditions this imitation can be produced through the destruction. In this case shapes are obtained by glass breaking (vandalism) but they can be used for constructive purposes (vandal-proof).

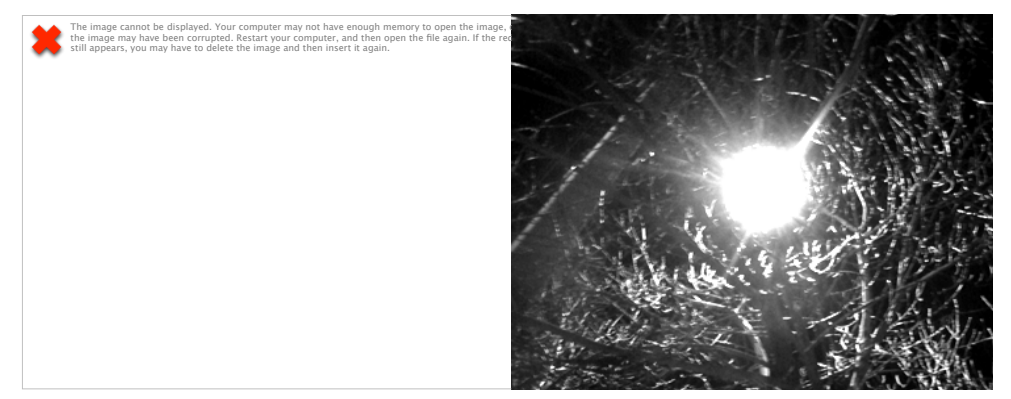

Fig. 5. A grid of cracks on the nonhardened glass obtained by a hit
Fig. 6. Optical phenomenon

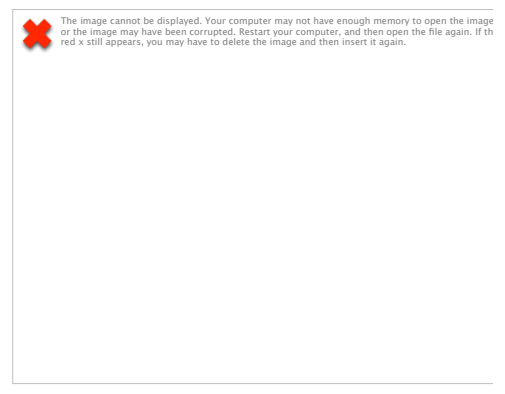

Fig. 7. A grid of cracks on the hardtempered glass 
The pattern with cracks of broken glass is used for decorating tables (Fig. 8). French designers painted the glass showcase to look like broken glass (Fig. 9), i.e. they used this form to control the attention of potential customers. However this advertising trick is risky in accordance with the results of studies of vandalism provoking factors. It was found that the initial small (trial) wrecking of the object provokes it more thorough destruction.

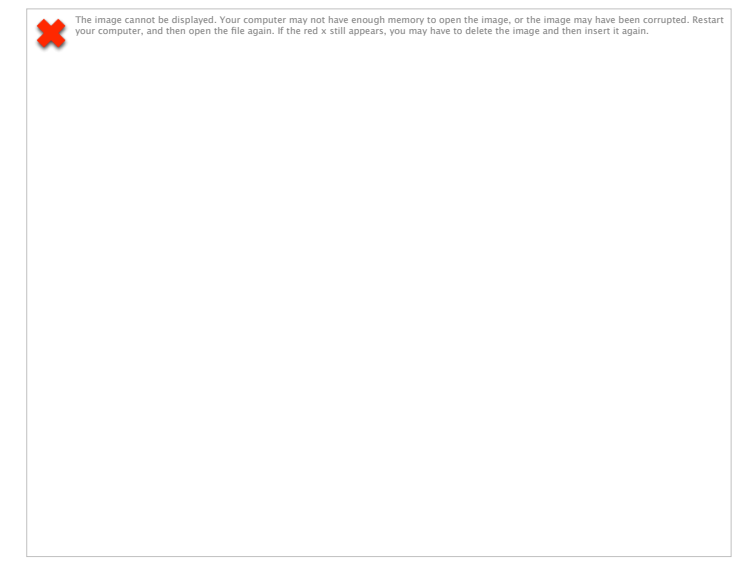

Fig. 8. The object decorated with cracks

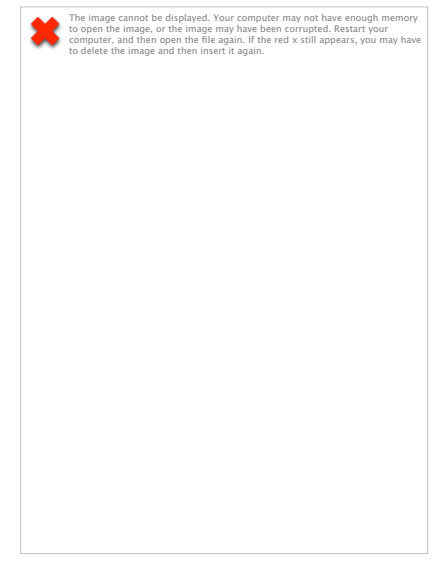

Fig. 9. Broken glass imitation

On the other hand, attention management is the sphere of information security for the society. It must be considered with taking into account objectives of social groups or a whole society. If the goal is the development of society, it is important to focus on how to point the attention of every society member into constructive sphere. At the same time it is necessary to expand the scope of creative forces application of every society member. The particular attention should be paid to people who are prone to meaningless vandalism. In this case, from a variety of motives vandalism can be highlighted as a desire to relieve irritation caused by the rigidity of any project frameworks. Here we go completely into the field of design. At this stage it is important to select a style. In the architecture an irritation from a disharmony of the buildings composition made in different styles is solved with the help of modernist style. In the interior design a goal of abirritation is achieved by using bionic style. From all the potential of this style we will allocate the thesis - principles of formatting the design objects should be similar to the constructive principles of biological objects. This thesis applies not only to the shape of objects but also to the principles of objects interaction.

In the biosphere there are no objects outside the interaction function. Here, all the elements are in the varying forms or degrees of interconnections. Roughly speaking, there is no waste in nature. Transferring this principle into the design sphere will give us the following two principles. Firstly, the production should be non-waste. Secondly, the garbage is a material for the construction with the appropriate technology. The first principle is implemented for a long time and it is the physical expression of the economic requirements (cost reduction). The implementation of the second principle has begun only after environmental awareness. Basically, what is garbage? It is actually a good thing being in the wrong place. This state is illustrated in Fig. 2. It is a useful thing (newsletters) in the wrong place. In fact, what we have is a lower bound of meaningless vandalism and the upper one is meaningful vandalism. Currently all secondary material is widely used to create an art object. Depending on author's skills, the resulting objects have a different aesthetic value. The importance here 
is not only in the use of recycled materials, but also in the goal of creating art objects. With an aim of the society (and each of its members) development, the compliance of the objectives should be verified in case of any art object.

If the production of objects is carried out to achieve the objectives that diverge with the purpose of society development, it will produce a lot of waste. It changes the perception of the physical and spiritual environment that can serve as a source of meaningless vandalism. In terms of ecology many technologies are senseless and in fact a kind of meaningless vandalism.

\section{Conclusion}

Due to space exploration image of the Earth as a sphere (closed space) has ceased to be scientific construct. Photos of the Earth led to the realization of all mankind that all processes in the world are interconnected. Local action leads to global consequences. The shape of the Earth (sphere) is not only the symbol of a space closure but also a symbol of the nonlinearity of the processes occurring on the Earth. Awareness of the interrelation leads to the conclusion that any action should be planned considering all relevant factors. Evaluation of significance should be based on awareness of the common purpose for all mankind. At the moment the overall objective is the development of humanity without destroying the environment. Violation of physical and spiritual ecology is the main source of meaningless vandalism. If resistance to vandalism is carried out by design, the creation of objects should be built considering the main goal - the development of society and each of its individual.

1. A meaningless and meaningful forms of vandalism cardinally differ by purpose.

2. Countering meaningful kind of vandalism is beyond the scope of design.

3. Activity of mindless vandalism can be reduced by means of design.

4. The design can both provoke and prevent the meaningless vandalism.

5. When designing aesthetically valuable objects, both stress and attention concentrators should be avoided. The object should have an outer appearance of physical durability.

6. The image of the destroyed object can be used to control the viewer's attention.

\section{References}

Allen, V., Greenberger, D. (1978). An aesthetic theory of vandalism.Crime and delinquency, 24 (3), 309-321 Allen, V., Greenberger, D. (1979). Enjoyment of destruction: The role of uncertainty. Journal of Nonverbal Behavior, 4(2) 87-96.

Goldstein, A. (1996). The psychology of vandalism. New York, NY: Plenum Press.

Kornienko, M., Kukhta, M. (2015). The experience of Visual Perception in the Design Education. Procedia Social and Behavioral Sciences, 206, 365-368

Kukhta, M., Sokolov, A. (2014). Welding technologies in art processing of metal. IOP Conference Series: Materials Science and Engineering, 66 (1) 1-5.

Plato. (1994). Hippias Major. Moscow, M: Misl'.

Sokolov, A., Kukhta, M. (2014). Modern technologies of decorative surface treatment. Mechanical Engineering, Automation and Control Systems: Proceedings of International Conference, 6(1) 1-4. doi: 10.1109/meacs.2014.6986951

Zhmurov, D. (2011). Glossary of aggression and violence. Saarbrücken, Sb: Lambert Academic Publishing 